\title{
Influence of size and morphology on the optical properties of carbon nanostructures
}

\author{
Hajar Sadeghi ${ }^{1} \cdot$ Davoud Dorranian $^{2}$ (i)
}

Received: 25 September 2015/ Accepted: 27 September 2015/Published online: 11 November 2015

(C) The Author(s) 2015. This article is published with open access at Springerlink.com

\begin{abstract}
Optical properties of carbon nanostructures produced by laser ablation method in different liquid environments have been studied experimentally. In this work we used a $7 \mathrm{~ns}$ fundamental wavelength of pulsed $\mathrm{Nd}$ :YAG laser at $1064 \mathrm{~nm}$ and $10 \mathrm{~Hz}$ repetition rate to produce carbon nanostructures including graphene in distilled water, acetone, alcohol, and CTAB. Structure and morphology of carbon nanostructures were studied using their UV-Vis-NIR spectrum, TEM images, and Raman scattering spectrum. Results show that in our experimental condition distilled water was the most capable medium for producing graphene. Color of suspensions varied with the amount of carbon nanoparticles in the suspension while the bandgap energy of samples decreases with increasing the amount of graphene.
\end{abstract}

Keywords Pulsed laser ablation · Carbon nanoparticles . TEM $\cdot$ Band gap energy $\cdot$ Graphene

\section{Introduction}

A wide range of electronic properties of carbon from insulating/semiconducting diamond to metal-like graphite, nanotubes and graphene sheets yields many technological applications in different areas of human activity [1]. In general, carbon-based materials play a major role in

Davoud Dorranian

doran@ srbiau.ac.ir

1 Physics Department, Science Faculty, Central Tehran Branch, Islamic Azad University, Tehran, Iran

2 Laser Lab., Plasma Physics Research Center, Science and Research Branch, Islamic Azad University, Tehran, Iran today's science and technology and the discovery of graphene is the last of a long string of continuous advances in the science of carbon [2]. Graphene is the experimental realization of a truly two-dimensional material [3]. Electrons in this two-dimensional crystal can be described by Dirac's equation, are fully relativistic, and exhibit extremely high mobility. Moreover, bi-layer graphene allows the fabrication of a field effect transistor with electric-fieldtunable band gap [4]. Carbon nanostructures and specially graphene with their extraordinary characteristics is expected to show fantastic effects in different field of optics as well. Incomparable electronic structure of graphene may open a new area in the interaction of electromagnetic wave with matter.

In this manuscript, we have investigated the optical effects due to the presence of graphene in the liquid suspension of different carbon nanostructures. Carbon nanostructures were produced by ablating a graphite plate with laser pulse in different liquid media. Usually in the ablation process the liquid medium in laser ablation process may determine the pressure of plasma plume which form on the surface of target in the ablation process [5]. Structure, size, and morphology of nanostructures may be affected strongly by the pressure of plasma plume. Regarding graphene production, penetration of the ablation liquid medium between graphite planes may lead to ablation of the plane structure of graphite, i.e. graphene. In this case the size of liquid molecules is important.

In our experiments we made different combinations of carbon nanostructures in different liquid media. Using absorption and transmission spectra of samples, their absorption coefficient, extinction coefficient, and bandgap energy were extracted and effect of carbon nanostructures especially graphene on them are discussed in detail. This work can be noticeable from two points of view. First is the 
behavior of carbon nanostructures against the UV-Vis-NIR spectrum of electromagnetic wave and second is the effect of carbon nanostructure morphology, i.e. nanoparticles or nano-sheets on this behavior.

This manuscript is organized as follows: following the introduction in Sect. 1 the experimental detail is described in Sect. 2. Section 3 is devoted to results and discussion and conclusion is presented in Sect. 4.

\section{Experimental}

Carbon nanostructures were produced by pulsed laser ablation of a graphite plate $(99.9 \%)$ in various liquid environments. A graphite plate was placed on the bottom of an open glass cylindrical vessel filled with $40 \mathrm{~mL}$ of liquid. Height of liquid on the target was $0.8 \mathrm{~cm}$. The graphite plate was cleaned ultrasonically in alcohol, acetone and deionized water before the experiments. Graphite target was ablated with the fundamental wavelength (1064 nm) of a pulsed Nd:YAG laser operated at $7 \mathrm{~ns}$ pulse width and $5 \mathrm{~Hz}$ repetition rate. 5000 laser pulse was used to produce carbon nanoparticles in various liquid environments. The fluence of laser beam was $1.5 \mathrm{~J} / \mathrm{cm}^{2}$ with $6 \mathrm{~mm}$ diameter. Output of laser was focused on the surface of graphite target using a $80 \mathrm{~mm}$ focal length convex lens.
The spot size of laser pulse on the surface of target was calculated to be $30 \mu \mathrm{m}$, leads to laser fluence of $60 \mathrm{~kJ} / \mathrm{cm}^{2}$ on the surface of graphite target. Samples 1 to 4 indicate the produced carbon nanostructures in distilled water, acetone, alcohol, and 0.01 molar Cetyl trimethylammonium bromide (CTAB), respectively. Details about the samples preparation are presented in Table 1.

A variety of analytical techniques were applied for the characterization of products. The optical properties of the nanoparticle solution were examined at room temperature by UV-Vis-NIR spectrophotometer from PG instruments Ltd. Transmission electron microscopy (TEM) was done by deposition a drop of the concentrated suspension on a carbon-coated copper grid. Raman Thermo Nicolet disperse spectroscope from Almega was utilized to determine the structure, quality and amount of graphene in dried drops of suspensions on $\mathrm{Si}$.

\section{Results and discussion}

Pictures of nanoparticle suspensions are presented in Fig. 1. They contain carbon nano-sheets, i.e. graphene and carbon nanoparticles. The first visual optical property of a material is its color. Color of carbon nanostructures solutions differs from dark brown to a colorless. Graphene is a
Table 1 Introduction and parameters of carbon nanostructures produced by laser ablation method in different liquids

Fig. 1 Carbon nanostructures produced by pulsed laser ablation in various liquid environments

\begin{tabular}{lllll}
\hline Sample number & S1 & S2 & S3 & S4 \\
\hline Liquid environment & Water & Acetone & Alcohol & CTAB 0.01 \\
Wavelength of absorption peak $(\mathrm{nm})$ & 290 & 304 & 271 & 262 \\
Band gap energy $(\mathrm{eV})$ & 2.80 & 3.50 & 3.95 & 4.48 \\
\hline
\end{tabular}

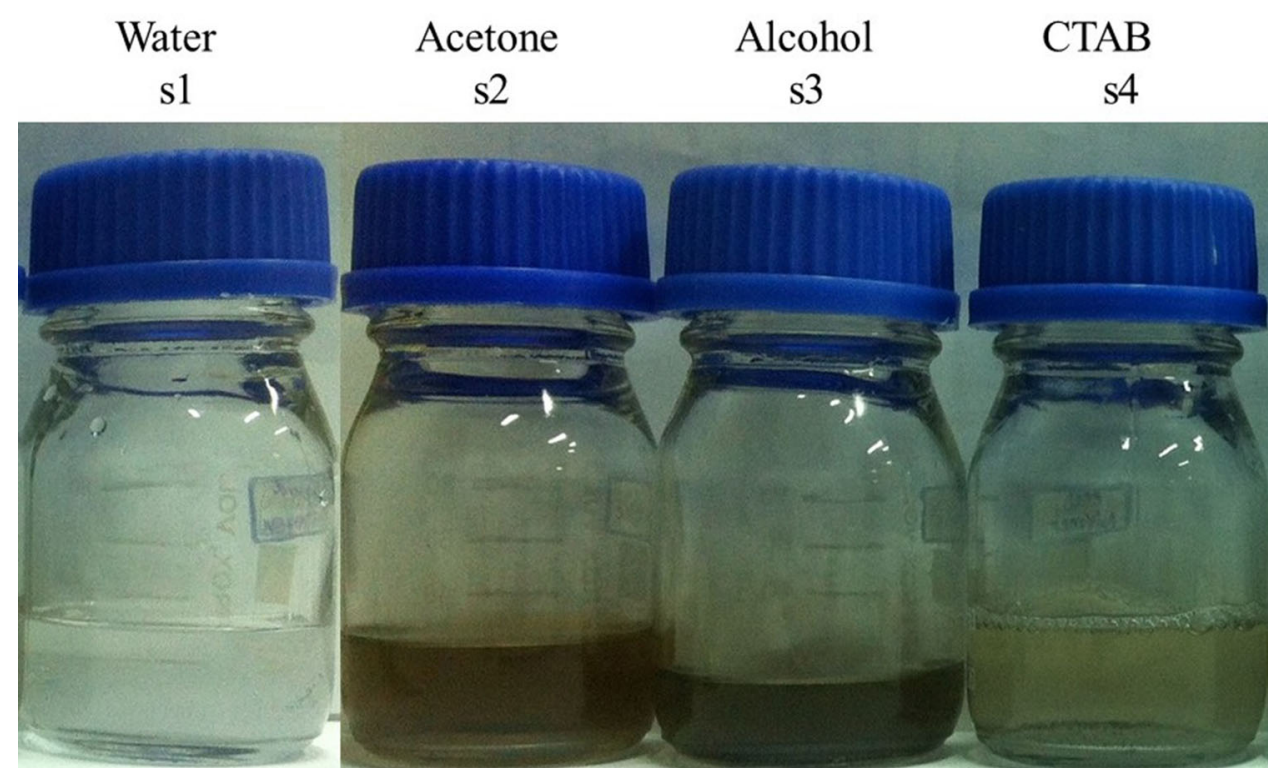


Fig. 2 Absorbance spectrum of carbon nanostructures suspensions in different liquids

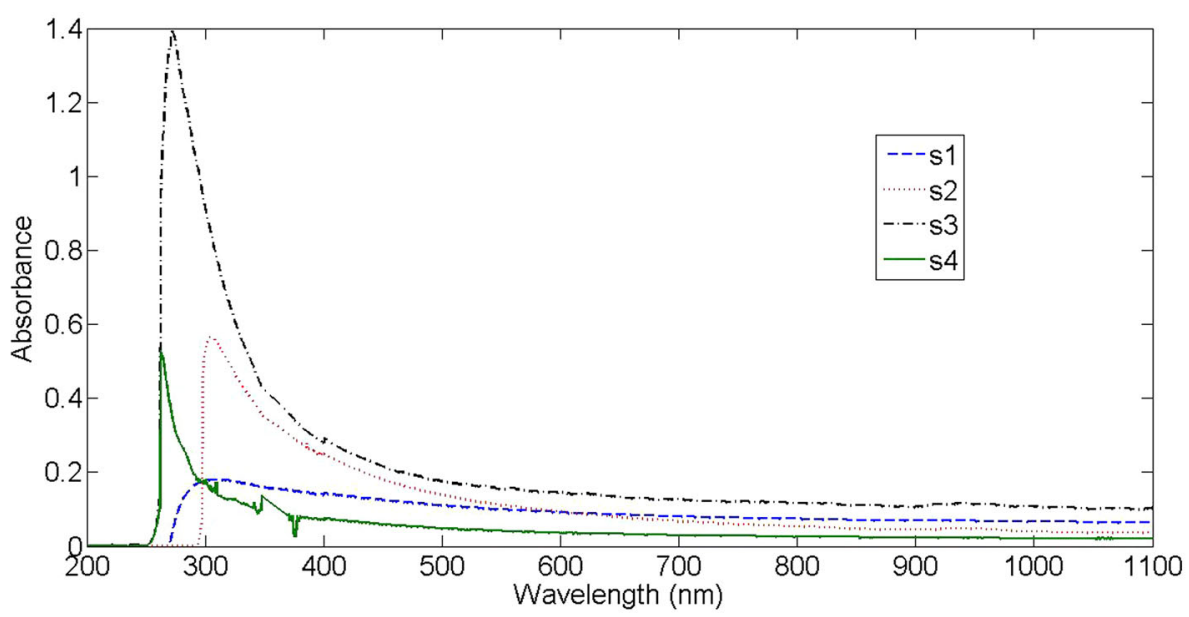

transparent and colorless sheet. In this case the color of suspensions may depend on the carbon nanostructure size, concentration, and morphology. As can be seen the color of carbon nanostructures in alcohol is darker than other liquids. After that from acetone to CTAB and water the color of solutions decreased, respectively.

To explain the reason, one may have a look at absorption spectrum of samples, TEM images, and Raman spectrum of samples which are presented in Figs. 2, 3, and 4 respectively.

The UV-Vis-NIR absorption spectra of samples in the $1 \mathrm{~cm}$ thickness quartz cuvette with the absorption of the liquid environment as the baseline are shown in Fig. 2. The absorption peak due to $\pi-\pi^{*}$ transition of $\mathrm{C}=\mathrm{C}$ band and $n-$ $\pi^{*}$ transition of $\mathrm{C}=\mathrm{O}$ band have been occurred around 250-300 nm [6, 7]. This absorption features are seen due to surface plasmon resonance (SPR) in the free electron cloud of carbonaceous material $\pi$ electrons [8]. The intensity of SPR peaks is proportional to the amount of carbon nanoparticles in the suspensions. The highest peak occurs due to carbon nanoparticles in alcohol. By decreasing the number of carbon nanoparticles in suspension, the SPR peaks of acetone and CTAB were decreased. And finally in the case of water as the ablation environment SPR peak is minimum. From the wavelength corresponding to SPR peak, we can compare the size of produced nanoparticles in different liquid media. The wavelength corresponding to SPR peaks of samples are indicated in Table 1. The SPR peak of nanoparticles is red shifted from CTAB to alcohol and to water and acetone. In other words, the size of the produced nanoparticles is increased from CTAB to alcohol and water and acetone. Although the laser wavelength, pulse width, and energy were the same for producing all samples, we have different carbon nanostructures in different ablation environments.

Morphology, size, and amount of produced carbon nanostructures can be studied using TEM images which are presented in Fig. 3. Data were recorded from the dried drop of suspensions on TEM grids. The number of carbon nanoparticles in water is minimum in comparison with other samples. After water, the number of carbon nanoparticles in CTAB and acetone is fewer than their number in alcohol, respectively. Furthermore in CTAB the aggregation of carbon nanoparticles are so high that we see them in a cluster like morphology, but in other liquids nanoparticles can be seen separately and the boundary between them is clear. The other carbon nanostructure which can be seen in TEM images is carbon nano-sheets. Bright color and transparency of nano-sheets in TEM images are what we exactly expect from graphene. The amount of graphene in CTAB is less than other samples. Produced nano-sheets in CTAB are small and condensed. The amount of nano-sheets increased in alcohol, acetone, and water.

The Raman spectra of samples in the range of $1200-1700 \mathrm{~cm}^{-1}$ are presented in Fig. 4. Data have been recorded from the dried drops of suspensions on silicon substrate with $0.1 \mathrm{~cm}^{-1}$ spectral resolution. The lines at 1354 and $1601 \mathrm{~cm}^{-1}$ are due to generated graphite and nanocrystalline graphite, respectively, while the $\mathrm{D}$ band at $1325 \mathrm{~cm}^{-1}, \mathrm{G}$ band at $1589 \mathrm{~cm}^{-1}$, and $\mathrm{D}^{\prime}$ band at $1618 \mathrm{~cm}^{-1}$ are attributed to the graphene in the samples. Similarly the lines at 137 and $1382 \mathrm{~cm}^{-1}$ are attributed to the amorphous carbons. In acetone and CTAB environments, Raman feature appears at about $1450 \mathrm{~cm}^{-1}$ and this line was assigned to be disordered carbons attributed to interstitial defects [1,9-11]. According to the Raman spectra, the intrinsic property of well-ordered $\mathrm{sp}^{2}$ carbons generated in CTAB is smaller than those created in the other liquid and amount of graphene in water, acetone and alcohol are larger than these sheets in CTAB environment.

So far we can conclude that the color of carbon nanostructure suspensions was due to concentration of carbon nanoparticles in the ablation environment and with 


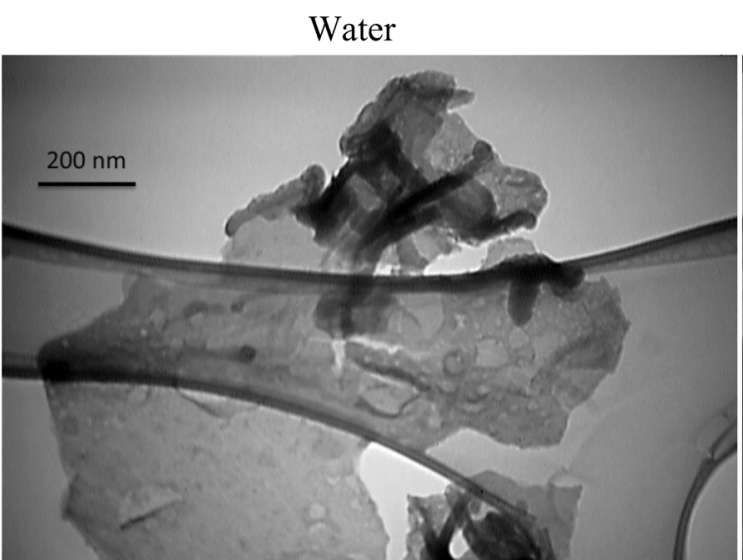

Alcohol

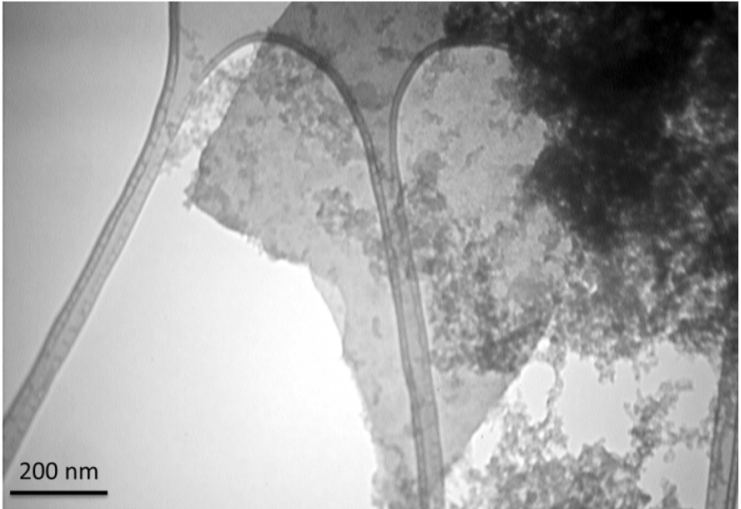

Fig. 3 TEM micrographs of samples
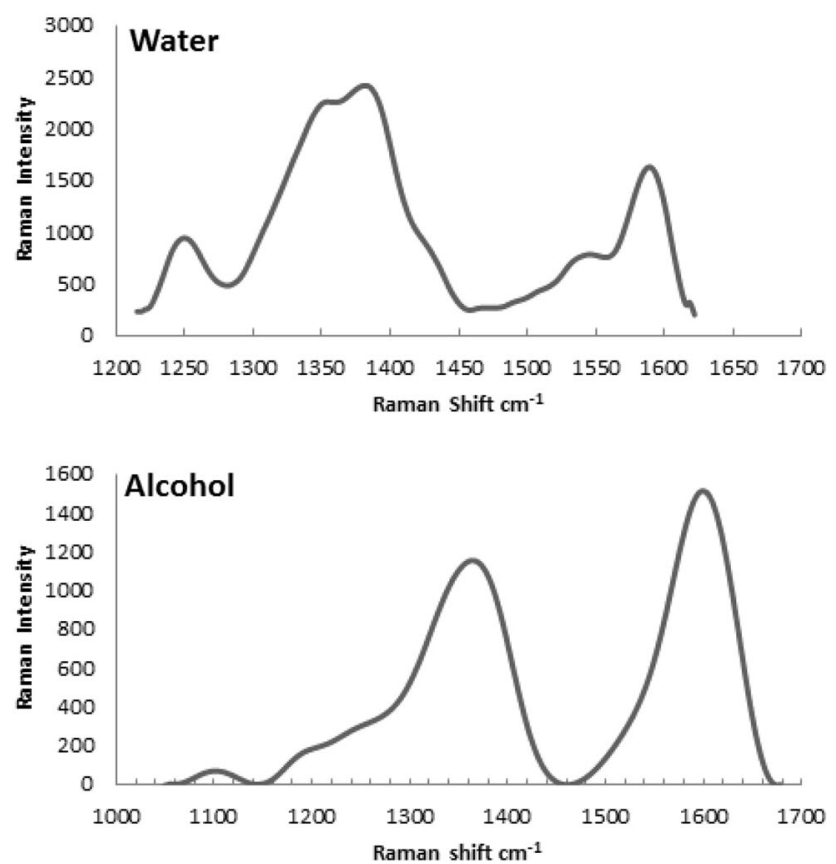

Fig. 4 Raman scattering spectrum of samples
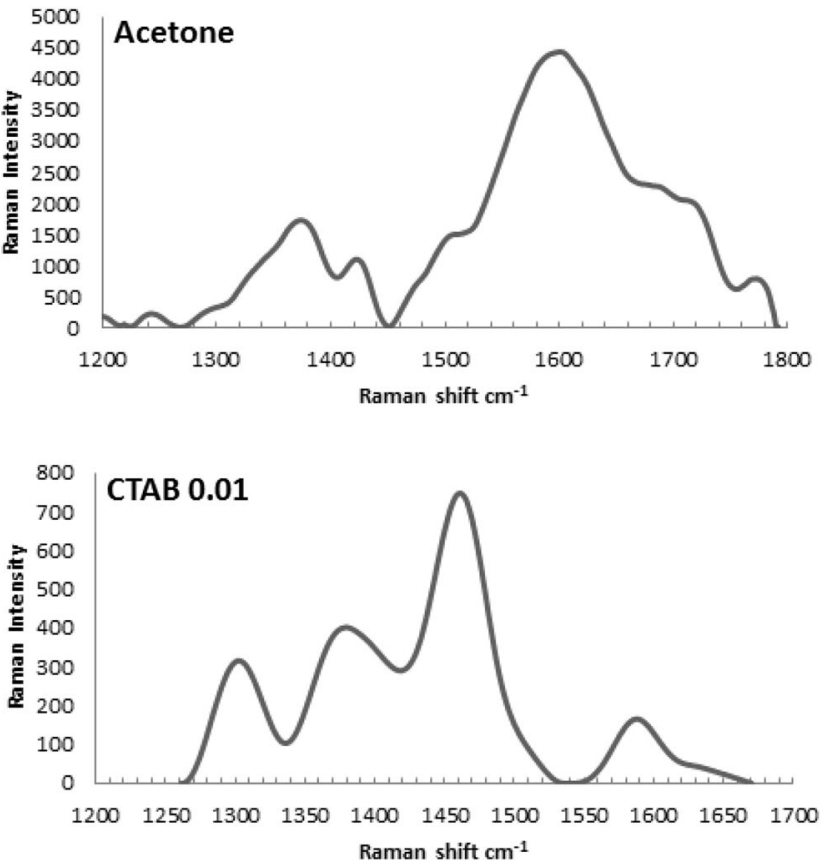
increasing the amount of carbon nanosheets, i.e. graphene in the suspension, it tended to a colorless fluid. By increasing the amount of graphene in the suspension, the intensity of SPR peak in the absorption spectrum decreased and the width of the peak increased.

The variation of transmittance $T$ as a function of wavelength for samples 1-4 were recorded at room temperature and are shown in Fig. 5. Sample 1 is the most transparent sample among these four. The absence of absorption edge can be seen only in the transmittance spectrum of this sample. The sharpest absorption edge was formed in sample 4 at wavelength $<300 \mathrm{~nm}$. The slope of absorption edge was reduced in samples 3 and 4, respectively. The sharp increase in the transmittance spectrum of samples $2-4$ is due to the presence of carbon nanostructures band gap. This figure clearly indicates that in the transmittance spectra of samples 2-4, a valley at $\lambda<300 \mathrm{~nm}$ occurred, that its intensity is different for different samples. The appearance of this valley is due to the surface plasmon resonance (SPR) nature of the carbon nanoparticles in the suspensions and its intensity depends on the amount of carbon nanoparticles in the suspensions. The results show that by increasing the amount of graphene in the suspensions the transmittance increases and absorption edge disappears.

The optical absorption coefficients of samples are evaluated from the transmittance data using [12]:

$\alpha=\frac{1}{d} \ln \left[\frac{1}{T}\right]$

where $\alpha$ is the absorption coefficient, $T$ is the transmittance, and $d$ is the thickness of samples, which was taken $1 \mathrm{~cm}$, equal to the thickness of quartz cuvette of spectrometer. Figure 6 presents the optical absorption coefficients of samples. Here also sample 1 with largest amount of graphene shows an exceptional behavior. Its absorption coefficient increased very slightly by increasing the photon energy and decreased to 0 when the energies of photons are larger than $4.3 \mathrm{eV}$. The absorption peaks at $4.75,4.65$, and $4.3 \mathrm{eV}$ for samples $4-2$ represent the characteristic surface
Fig. 5 Transmission spectrum of carbon nanostructure suspensions in different liquids

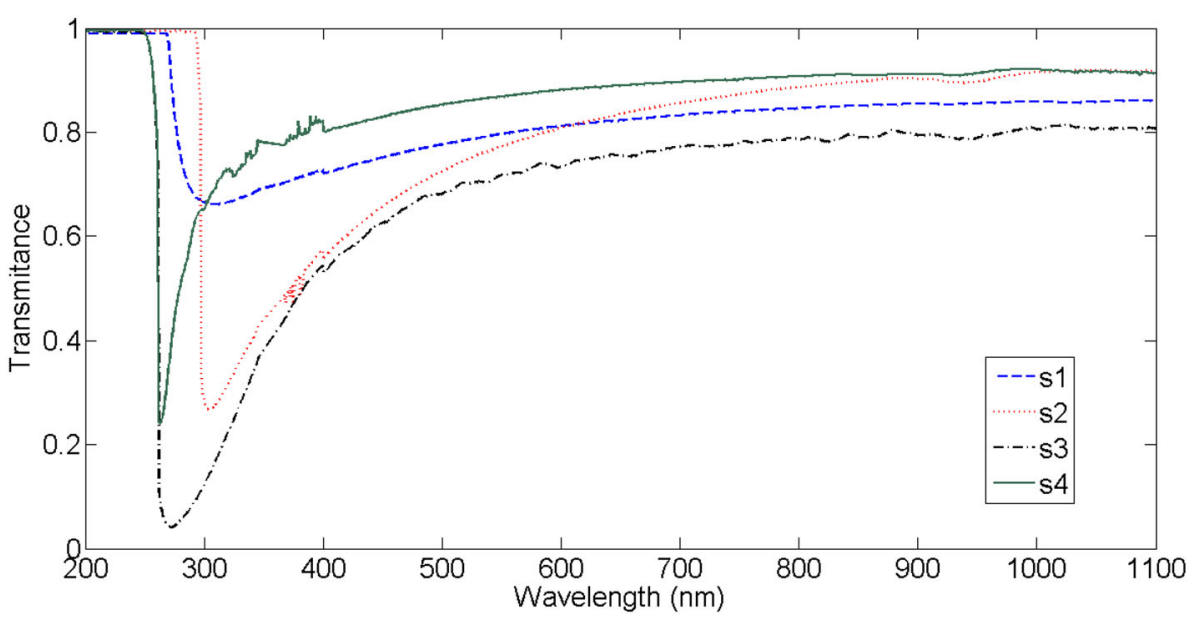

Fig. 6 Absorption coefficient of carbon nanostructure suspensions in different liquids

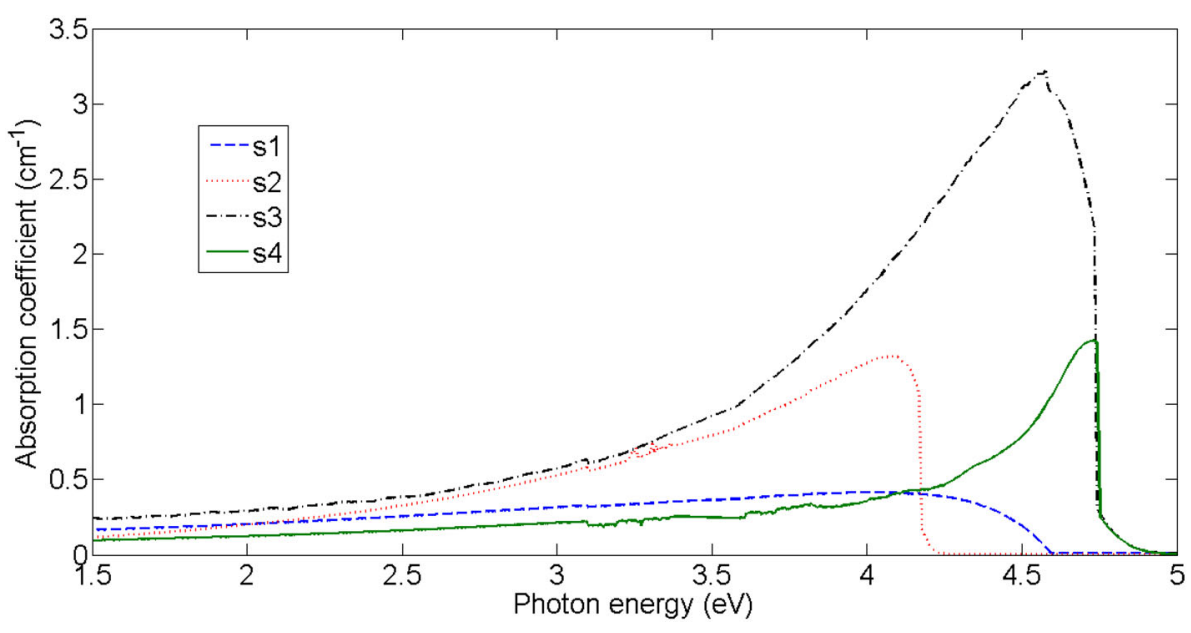


Fig. 7 Illustration of Tauce method to extract the band gap energy of samples. Bandgap energy is the point at which the tangent line to the absorption edge (black line) extrapolates with the photon energy axes
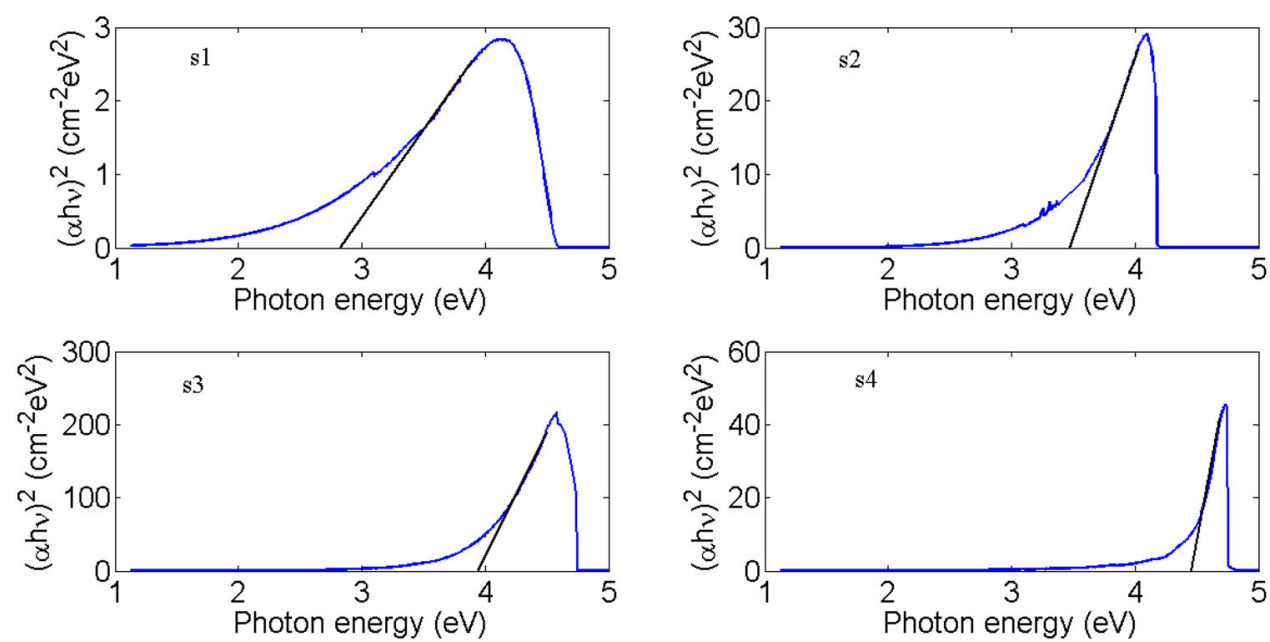

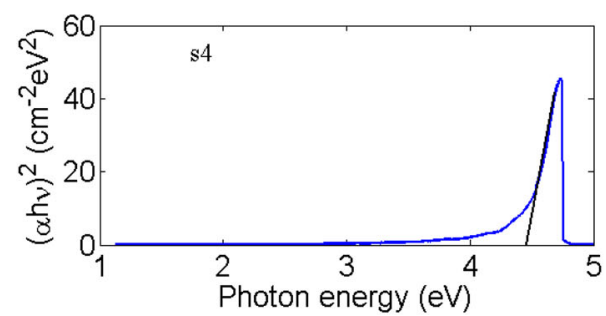

Plasmon resonance dedicated to carbon nanoparticles. The presence of nanoparticles in the suspensions could be conveniently followed by monitoring the Plasmon absorption peaks in the absorption spectrum. The larger absorption peak, appeared in UV range, is due to the energy gap of samples in this area. The position of the absorption edge was determined by extrapolating the linear portions of $\alpha$ versus $h v$ curves to zero absorption value. It is clear from Fig. 6 that the band edge showed a decrease with increasing the size of carbon nanoparticles in the suspensions. The absorption edge shifted towards higher wavelength side, indicating the decrease in the optical band gap for the samples. Thus, shift of the absorption edge in the UV region, is due to changes in the electron-hole in the conduction and valence bands.

The most used method for the estimation of the band gap from optical measurement is the one proposed by Tauce [13]. The optical band gap of samples was deduced from the intercept of the extrapolated linear part of the plot of $(\alpha E)^{2}$ versus the photon energy $E$ with abscissa (see Fig. 7). This follows by the method of Tauce where:

$\alpha E=B\left(E-E_{\mathrm{g}}\right)^{p}$

In this equation, $\alpha$ is the absorption coefficient, $E$ is the photon energy, $B$ is a factor that depends on transition probability and can be assumed to be constant within the optical frequency range and the index $p$ is related to the distribution of the density of states is an index which assumes the values $1 / 2,3 / 2,2$ and 3 depending on the nature of electronic transition. Taking $p=1 / 2$, which corresponds to directly allowed transition in carbon nanoparticles; the band gap energies of samples were calculated. In a direct gap, a photon can be emitted because the electron must pass through an intermediate state without transferring momentum to the crystal lattice. Bandgap energies of samples are presented in Table 1. As can be seen, the energy gap of samples is increased noticeably from sample 1 to $4 . \quad E_{\mathrm{g}} \quad(\mathrm{S} 4)=4.48 \mathrm{eV}, \quad E_{\mathrm{g}}$ $(\mathrm{S} 3)=3.95 \mathrm{eV}, E_{\mathrm{g}}(\mathrm{S} 2)=3.5 \mathrm{eV}$, and $E_{\mathrm{g}}(\mathrm{S} 1)=2.8 \mathrm{eV}$ is reduced. Actually by increasing the amount of graphene in the suspension, the bandgap energy decreases. In the case of samples 2-4 the magnitude of bandgap energy is inversely proportional to the size of carbon nanoparticles, which is a general rule. Variation of bandgap energy of samples is in very good agreement with the results of TEM images and their absorption spectrum SPR peaks, as well as Raman data. By increasing the amount of graphene in the suspensions, the band gap energies of samples is decreased.

\section{Conclusion}

With the aim of understanding the condition to produce graphene by laser ablation method, the effect of graphene amount on the optical properties of carbon nanostructures was investigated experimentally. Raman data and TEM images indicate that the amount of produced graphene sheets in water was the highest in comparison with other samples. This amount decreased in acetone, alcohol, and CTAB. The intensity of absorption peaks confirms that the amount of carbon nanoparticles is maximum in alcohol and decreased in acetone, CTAB, and water. The wavelength corresponds to SPR peaks show that the largest carbon nanoparticles are produced in acetone and their size decreased in water, alcohol, and CTAB. In this case the color of suspensions is due to the amount of carbon nanoparticles and graphene in the suspension, while the bandgap energy of nanostructure strongly depends on the amount of graphene in the suspensions.

Open Access This article is distributed under the terms of the Creative Commons Attribution 4.0 International License (http://crea tivecommons.org/licenses/by/4.0/), which permits unrestricted use, 
distribution, and reproduction in any medium, provided you give appropriate credit to the original author(s) and the source, provide a link to the Creative Commons license, and indicate if changes were made.

\section{References}

1. Mortazavi, S.Z., Parvin, P., Reyhani, A., Mirershadi, S., Sadighi Bonabi, R.: Generation of various carbon nanostructures in water using IR/UV laser ablation. J. Phys. D Appl. Phys. 46, 165303 (2013)

2. Ferrari, A.C.: Raman spectroscopy of graphene and graphite: disorder, electron-phonon coupling, doping and nonadiabatic effects. Solid State Commun. 143, 47-57 (2007)

3. Proctor, J.E., Gregoryanz, E., Novoselov, K.S., Lotya, M., Coleman, J.N., Halsall, M.P.: High-pressure Raman spectroscopy of graphene. Phys. Rev. B 80, 073408 (2009)

4. Dhar, S., Barman, A.R., Ni, G.X., Wang, X., Xu, X.F., Zheng, Y., Tripathy, S., Rusydi, A.A., Loh, K.P., Rubhausen, M., Castro Neto, A.H., Zyilmaz, B.O., Venkatesan, T.: A new route to graphene layers by selective laser ablation. AIP Adv. 1, 022109 (2011)

5. Moradi, M., Solati, E., Darvishi, S., Dorranian, D.: Effect of aqueous ablation environment on the characteristics of $\mathrm{ZnO}$ nanoparticles produced by laser ablation. J. Clust. Sci. 13, 1-12 (2015)
6. Chen, J.L., Yan, X.P.: A dehydration and stabilizer free approach to production of stable water dispersions of graphene nanosheets. J. Mater. Chem. 20, 4328-4332 (2010)

7. Kumar, V., Singh, V., Umrao, S., Parashar, V., Abraham, Sh, Singh, A.K., Nath, G., Saxena, P.S., Srivastava, A.: Facile, rapid and upscaled synthesis of green luminescent functional graphene quantum dots for bioimaging. RSC Adv. 4, 21101-21107 (2014)

8. Ghanbari, H., Sarraf-Mamoory, R., Sabbaghzadeh, J., Chehrghani, A., Malekfar, R.: Nonlinear optical absorption of carbon nanostructures synthesized by laser ablation of highly oriented pyrolytic graphite in organic solvents. Int. J. Opt. Photonics 7, 113-124 (2013)

9. Mortazavi, S.Z., Parvin, P., Reyhani, A.: Fabrication of graphene based on Q-switched Nd:YAG laser ablation of graphite target in liquid nitrogen. Laser Phys. Lett. 9, 547-552 (2012)

10. Ferrari, A.C., Meyer, J.C., Scardaci, V., Casiraghi, C., Lazzeri, M., Mauri, F., Piscanec, S., Jiang, D., Novoselov, K.S., Roth, S., Geim, A.K.: Raman spectrum of graphene and graphene layers. Phys. Rev. Lett. 97, 187401 (2006)

11. Lee, Y., Yamada, M., Miyake, M.: Synthesis of carbon nanotubes and carbon nanofilaments over palladium supported catalysts. Sci. Technol. Adv. Mater. 6, 420-426 (2005)

12. Alias, S.S., Mohamad, A.A.: Synthesis of Zinc Oxide by Sol-Gel Method for Photoelectrochemical Cells. Springer Science \& Business Media, NewYork (2014)

13. Tauc, J., Grigorovici, R., Vancu, A.: Optical properties and electronic structure of amorphous germanium. Phys. Stat. Sol. 15, 627 (1996) 